\title{
UN LETTO FUNEBRE CON DECORAZIONE IN OSSO DA TERAMO (ITALIA)
}

\author{
POR
}

\author{
MARIA GRAZIA D'AGATA E SILVIA BARBETTA \\ Università degli Studi di Roma «La Sapienza»
}

\section{RESUMEN}

Las autoras proponen la reconstrucción morfológica e iconográfica de la decoración en hueso de un lecho funerario romano a partir de numerosos fragmentos de revestimiento procedentes de una sepultura in bustum de la necrópolis de La Cona en Teramo (Abruzzo). El lecho, integrado en la tipología del mobiliario con "patas torneadas", estaba probablemente adornado con un tema báquico y se encuadra cronológicamente en la primera mitad del siglo I d.C., época de mayor difusión de este tipo de muebles.

\section{SUMMARY}

The authors propose a morphological and iconographic reconstruction of a Roman funeral couch decorated in bone working upon numerous cladding pieces coming from a grave in bustum in the necropolis of La Cona - Teramo (Abruzzo). The bed, classified within the «turned legs» typology, was probably adorned with a Bacchic iconographic theme and can be dated on the first half of the Ist century A.D., the time that records the widest diffusion of this kind furniture.

\section{SCOPERTA E DESCRIZIONE DEGLI ELE- MENTI \\ Per Maria Grazia d’Agata}

\section{INTRODUZIONE}

Nel settembre del 1984, una situazione di emergenza dovuta a lavori per la costruzione di un parcheggio nell'area sottostante il viadotto di Ponte Messato, sulla S.S.80, in località Madonna della Cona, costrinse la Soprintendenza Archeologica d'Abruzzo, in collaborazione con la Cattedra di Topografia ed Urbanistica dell'Università di Roma La Sapienza, ad intervenire con una campagna di scavo nella zona interessata ${ }^{1}$.

Era infatti venuta alla luce una nuova area della necropoli, già scavata nel 1961 dall'allora soprin-

\footnotetext{
1 Un sentito ringraziamento va alla Dr.ssa Luisa Migliorati, direttrice dello scavo, per aver permesso lo studio e la pubblicazione del materiale osseo ed alla Dr.ssa Giovanna Germana Grita per i preziosi consigli forniti.
}

tendente A. La Regina ${ }^{2}$, topograficamente connessa all'antico asse viario di collegamento tra l'antica Interamnia Praetuttiorum e l'hinterland abruzzese ${ }^{3}$. La necropoli comprende tombe a tumulo, a fossa e alla cappuccina, rinvenute nella zona retrostante una serie di sepolcri di età romana, distribuiti lungo l'asse viario che dava accesso, da est, alla città; l'utilizzo dell' area, dove sono contestualmente testimoniati i riti dell'incinerazione e dell'inumazione, copre un arco cronologico che va dal Ix sec. a.C. al i sec. d.C. Delle tombe monumentali di età romana rimangono i soli basamenti a pianta circolare, quadrata o rettangolare, costituiti da ciottoli di fiume legati da malta e rivestiti da grossi blocchi squadrati di travertino locale (fig. 1).

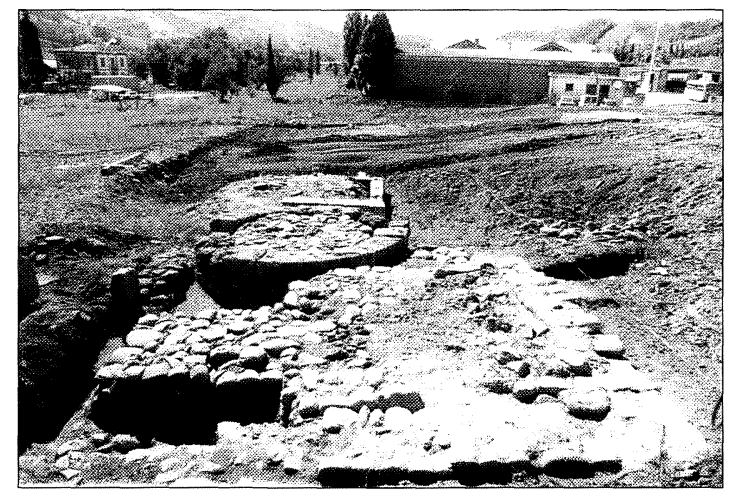

Fig. 1.-Panoramica della necropoli romana.

Oggetto dello scavo fu una tomba a pianta quadrangolare, di m. $3 \times 2,50$, con avancorpo prospiciente la strada (fig. 2), che ha restituito, tra l'altro, frammenti di osso lavorato, relativi ad un letto funebre. Al momento dell'intervento il sepolcro risulta-

${ }^{2}$ Ulteriori interventi si ebbero nel 1973 e nel 1990. W. Mazzitti, Teramo Archeologica, Teramo 1983, p. 157.

${ }^{3}$ Per questa viabilità, identificata con la via Caecilia, v. M. P. Guidobaldi, La Via Caecilia: considerazioni sulla cronologia e sul percorso di una via publica romana, in corso di stampa; S. Barbetta, La via Caecilia da Roma ad Amiternum, in c.s. 


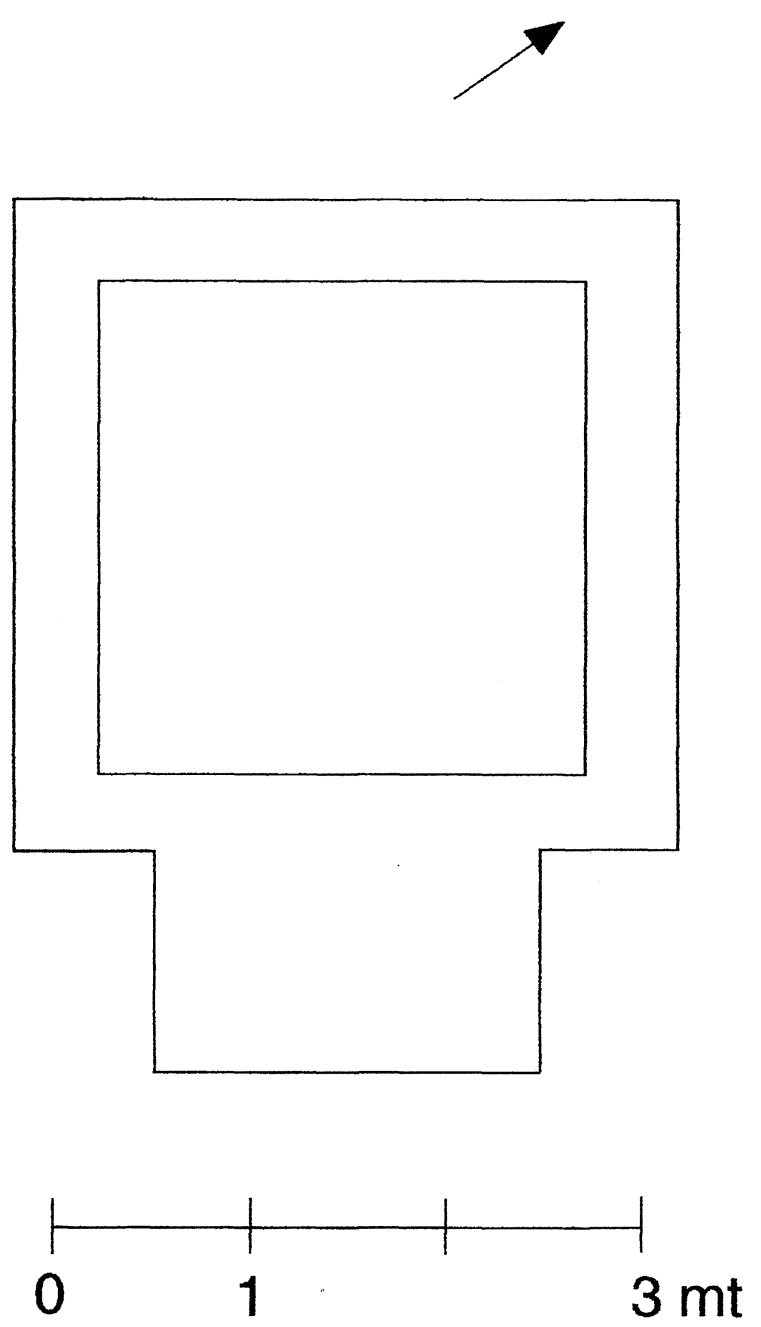

Fig. 2.-Rilievo schematico della tomba.

va parzialmente intaccato dai moderni lavori di sbancamento; i frammenti d'osso e quelli del corredo ceramico erano caoticamente disposti nell'interno, sconvolto dalla pala meccanica.

Tuttavia il successivo approfondimento degli strati ha consentito di rilevare una prevalente concentrazione degli elementi ossei al centro della tomba, mentre i frammenti ceramici sono stati ritrovati, per la maggior parte, lungo il lato interno del muro occidentale. Si tratta in prevalenza di frammenti di lucerne, oinochoai ed olle in ceramica depurata, di piatti in sigillata italica e balsamari in vetro ${ }^{4}$. Lo scavo ha evidenziato, in corrispondenza dei frammenti ossei, una compatta lente di bruciato dalla forma allungata, circoscritta da un piano omogeneo di

${ }^{4}$ Tutto il materiale è stato consegnato alla Soprintendenza Archeologica di Chieti per il restauro. ciottoli di fiume; al di sotto, un altro piano di ciottoli ricopriva tutta l'area della tomba. Anche il materiale presentava evidenti tracce di combustione, causa principale della lacunosità e dello strato di estremo deperimento nel quale sono stati ritrovati $\mathrm{i}$ frammenti ossei.

Si è accennato alla avvenuta manomissione della tomba e dunque alla possibilità che il cattivo stato del rinvenimento e l'esiguità del rivestimento osseo e degli altri elementi costitutivi del letto fossero attribuibili ad un evento moderno. E' però possibile che la situazione fosse già compromessa dalle conseguenze di un rituale funebre ben conosciuto: la presenza dello strato compatto di bruciato, dalla forma oblunga, all'interno della tomba, suggerisce la presenza di un bustum, una buca ricavata nella tomba stessa e destinata al rogo, con la successiva copertura dei resti in loco ${ }^{5}$. Tale procedimento causò la totale frammentazione della decorazione del letto e la calcinazione dell'osso utilizzato, determinandone la distruzione nei casi delle lavorazioni di minor spessore. E' probabilmente per questo motivo che sono meglio conservati gli elementi portanti del letto che non le decorazioni pertinenti i fulcra ed il telaio, consistenti in sottili lamelle decorate.

Si può inoltre osservare come l'entità del ritrovamento di materiale pertinente un letto funebre non sia poi così differente nel caso delle incinerazioni in busta rispetto a quelle in ustrina, risultando devastante, in entrambi i casi, l'azione del fuoco. Tuttavia mentre nel primo caso si possono ricostruire gli elementi rimasti come facenti parte di un'unica struttura, nel secondo esiste la possibilità che a ciò che era stato bruciato con il defunto si aggiungessero elementi derivati da una precedente cremazione.

I dati di scavo e le considerazioni fin qui esposte, dunque, attestano la presenza di una sola deposizione all'interno della tomba. L'impossibilità di poter disporre, per i motivi accennati, della totalità del rivestimento osseo, ha consentito uno studio limitato solo ad alcuni degli elementi del rivestimento e ha permesso una ricostruzione solo indicativa del letto funebre, assimilabile ai tipi conosciuti di Amiternum e di Cambridge.

\section{GLI ELEMENTI}

$$
\text { A.-GAMBA }
$$

A1. Puntale costituito da tre frammenti ricavati da un unico osso tubolare: h. cm. 2 ; spess. cm. 0,03; diam. max. cm. 2; diam. min. cm. 1,4

\footnotetext{
${ }^{5}$ Su tale rito cfr. Letta 1984, p. 94.
} 
A2. Anello a profilo rettilineo e a sezione piena: spess. cm. 0,4; diam. max. cm. 5; diam. min. cm. 1,4.

A3. Puntale con incassi interni ai bordi ricavato da un unico osso tubolare. Restano tre frammenti: h. cm. 3; spess. cm. 0,3; diam. max. cm. 2; diam. $\min . \mathrm{cm} .1,4$.

A4. Anello troncoconico con bordo profilato; ne restano tre frammenti: spess. cm. 0,3; diam. max. cm. 5; diam. min. cm. 1,4 (fig. 3).

A5. Anello troncoconico con profilo comprendente una metà concava ed una convessa, separate da un dente che crea una profilatura orizzontale. $\mathrm{Ne}$ resta un frammento: spess. cm. 0,3; diam. max. $\mathrm{cm}$. 5; diam. min. cm. 1,4.

A6. Strozzatura ricavata da un unico osso tubolare, con modanatura ai margini inferiore e superiore. Ne restano quattro frammenti: h. cm. 1,2; spess. cm. 0,2 ; spess. in corr. delle modanature cm.1,4; diam. max. cm. 2,2; diam. min. cm. 1,4.

A7. Anello a profilo leggermente concavo con incasso interno. Ne restano due frammenti: spess. cm. 0,3 ; diam. max. cm. 4,5; diam. min. cm. 1,4 (fig. 4).

A8. Anello troncoconico con bordo profilato, compostao da più lamelle. Ne restano quattro frammenti: h. cm. 3,8; spess. cm. 0,3; diam. max. cm. 6; diam. min. cm. 1,4.

A9. Strozzatura ricavata da un unico osso tubolare, con modanatura ai margini inferiore e superiore. Ne restano tre frammenti: h. cm. 1,2; spess. cm. 0,2 ; spess. in corr. delle modanature $\mathrm{cm} .1,4$; diam. max. cm. 2,2; diam. min. cm.1,4.

A10. Variante di A8. Ne restano sei frammenti: spess. cm. 0,3; diam. max. cm. 6; diam. min. cm.1,4 (fig. 5).

A11. Variante di A7. Ne resta un frammento: h. cm. 3,8 (fig. 6). Spess. cm. 0,3; diam. max. cm. 4; diam. min. cm. 1,4.

A12. Variante di A9. Ne restano due frammenti: h. cm. 1,4; spess. cm. 0,3; spess. in corr. della modanatura cm. 0,4; diam. max. $\mathrm{cm} .2,3$; diam. min. cm. 1,4 (fig. 7).

A13. Anello a profilo arrotondato costituito da più lamelle decorate con sottili strigilature: h. $\mathrm{cm}$. 2,5; spess. cm. 0,2; diam. max. cm. 6; diam. min. $\mathrm{cm} .1,4$. Gli elementi rimasti permettono la identificazione di tre anelli formanti un toro (fig. 8).

A14. Variante di A9: h. cm.1,4; spess. cm. 0,3; spess. in corr. delle modanature cm. 0,5; diam. max. cm. 2,5; diam. min. cm. 1,4 (fig. 9).

A15. Variante di A3. Ne resta un frammento: h. cm. 4,2; spess. cm. 0,3; diam. max. cm. 2,4; diam. min. cm. 1,4 .
A16. Anello troncoconico con bordo profilato. Ne restano 9 frammenti: spess. cm. 0,3; diam. max. cm. 6, diam. min. cm. 1,4 (fig. 10).

A17. Anello troncoconico a profilo rettilineo con gola presso il margine superiore e due listelli orizzontali; in origine composto da più elementi, ne rimangono quattro: h. cm. 4,5; spess. cm. 0,3 ; spess. in corr. delle modanature $\mathrm{cm} .0,5$; diam. $\max \mathrm{cm}$. 10; diam. min. cm. 2.

A18 e A22. Cilindro figurato su due registri.

A 21, A23, A26 (fig. 11):

- Amorino citaredo in corsa verso sinistra, ricostruito da tre frammenti: h. cm. 3,2; largh. cm. 2,4; spess. cm. 0,4.

- Amorino citaredo in corsa verso sinistra. Manca totalmente la gamba destra e parte della lira: $h$. cm. 3,3; largh. cm. 2,6; spess. cm. 0,8.

- Amorino citaredo in corsa verso destra. Ala e lira spezzate, mancante del piede sinistro: h. $\mathrm{cm}$. 3,3 ; largh. cm. 2,8; spess. cm. 0,8 .

- Amorino citaredo in corsa verso destra. Manca di parte dell'ala: h. cm. 2,5; largh. cm. 2; spess. cm. 0,5 .

- Amorino citaredo in corsa verso destra. Manca della lira: h. cm. 3,3; largh. cm. 2,8; spess. cm. 0,8 .

A31. Figura acefala e priva di braccia, panneggiata, su piedistallo modanato: h. cm. 4,8; largh. cm. 1,5 ; spess. cm. 0,5 .

A32. Busto acefalo di figura maschile ammantata, con braccio flesso internamente verso l'alto. Mancante del braccio sinistro: h. cm. 2,7; largh. cm. 1,7; spess. cm. 0,6 (fig. 12).

A33. Figura acefala panneggiata e stante su piedistallo modanato; mano sinistra al petto, mancante della parte destra: h. cm. 5,3; largh. cm. 1,5; spess. medio $\mathrm{cm}$. 0,7 (fig. 13).

A34. Variante di A9 con modanatura solo in corrispondenza del margine superiore: h. cm. 0,8 ; spess cm. 0,2 ; spess. in corr. della modanatura $\mathrm{cm}$. 0,4; diam. max cm. 2,3; diam. min. cm. 1,4.

A35. Fascia anulare troncoconica a lamina sottile con decorazione ad ovoli (kymation lesbico) con sottile cornice a fascia nella parte inferiore: $\mathrm{h} . \mathrm{cm}$. 0,8 ; spess. cm. 0,2; diam. max. cm. 2; diam. min. cm. 14 .

A36. Anello troncoconico con bordo arrotondato e dente che crea una profilatura orizzontale: spess. cm. 0,3; diam. max. cm. 7; diam. min. cm. 1,4.

A37. Anello troncoconico con profilo modanato e bordo a mandorla; presenta un incasso sul bordo superiore, probabilmente per alloggiare una borchia: spess. cm. 0,3; diam. max. cm. 7; diam. min. cm. 3 . 


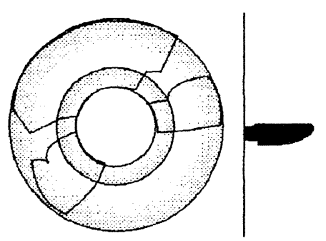

3

A 4

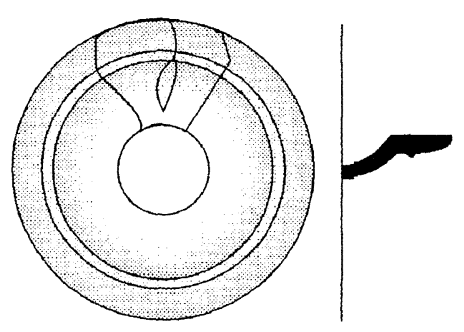

$4 \quad$ A 7

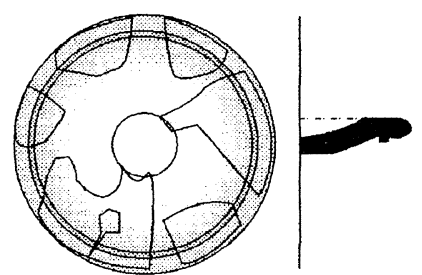

5

A 10

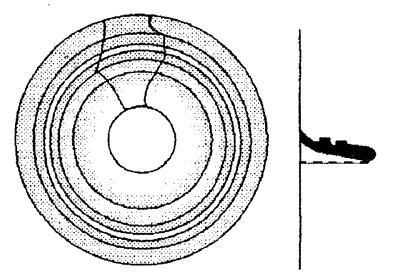

A 11

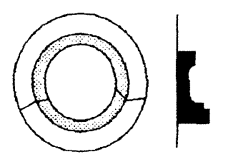

A 12 7

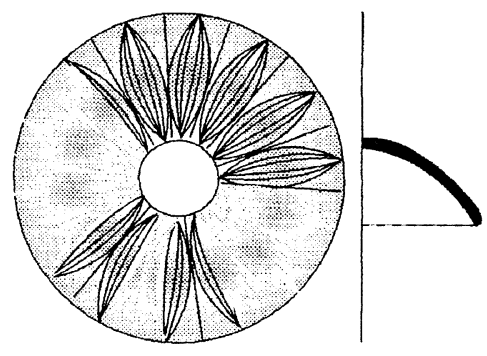

A 13

8

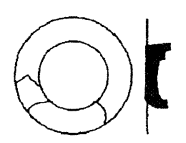

A 14

9

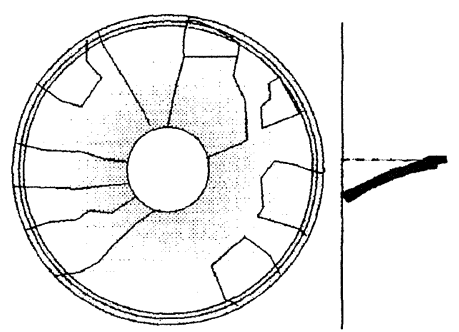

A 16

10

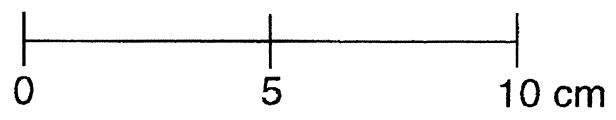

Figg. 3 a 10.-3-6, 8, 10: anelli modanati; 7, 9: strozzature.

A38. Borchia lenticolare con foro centrale (per il fissaggio tramite chiodino); presenta un dente sul bordo che crea una profilatura orizzontale; incompleta: spess. cm. 0,4; diam. cm. 3 .

\section{B.-TELAIO}

B1. Cornice a bastoncello semicilindrico del tipo ad astragali; ne restano due frammenti: cm. 2 e 


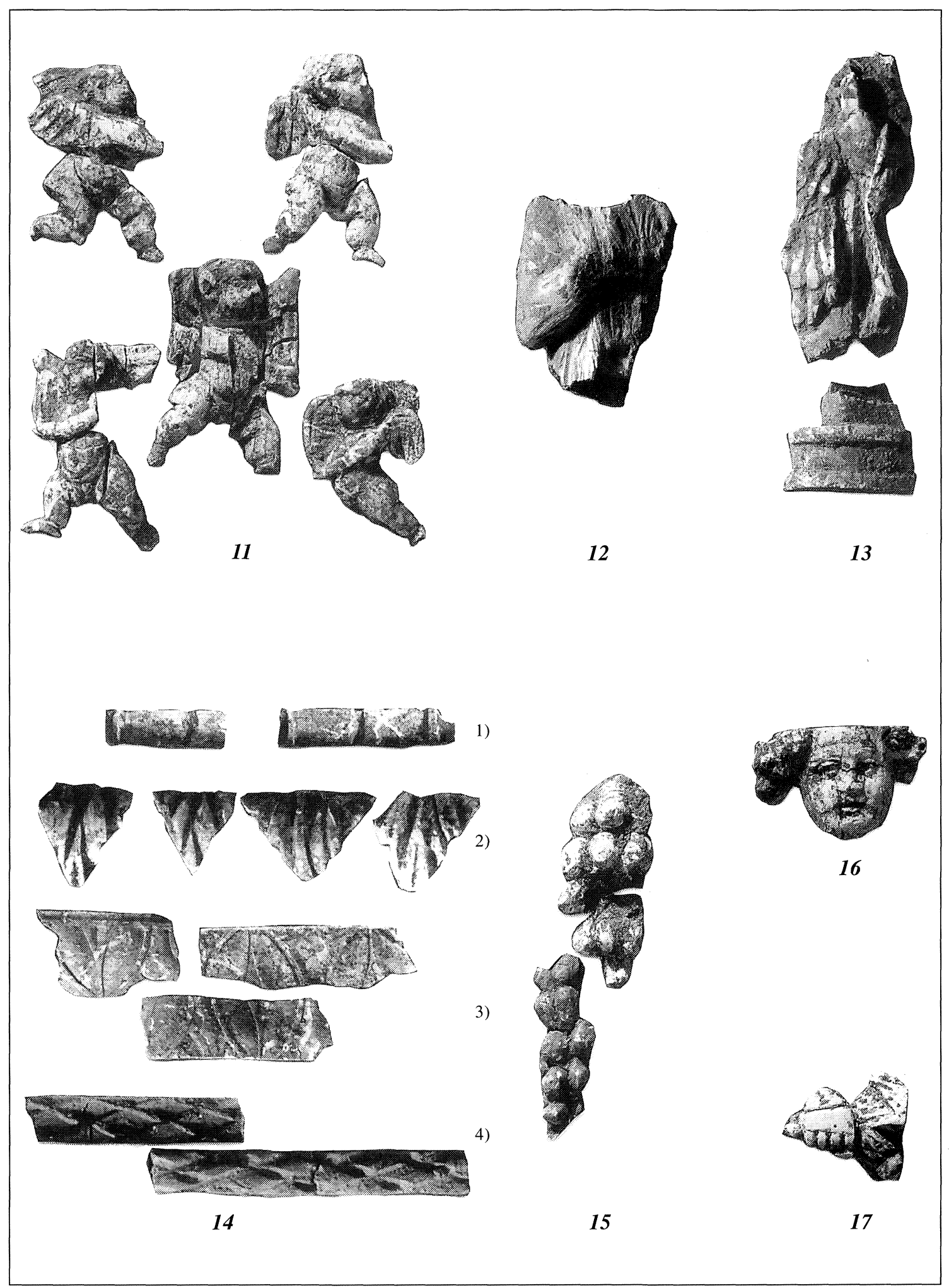

Figg. 11 a 17.-11: amorini citaredi; 12: busto acefalo; 13: figura acefala su base modanata; 14: elementi della cornice del telaio; 15: frammento di grappolo d'uva; 16: volto; 17: mano sinistra stringente il panneggio. 
3 di lungh.; largh. cm. 0,6; spess. cm. 0,4 (fig. 14.1).

B10. Lamella a lingua ricurva decorata a foglia d'acanto, ricavata da un unico osso: $h$. cm. 1,5; largh. cm. 2,6; spess. cm. 0,4. Ne restano 7 frammenti, tutti circa delle stesse dimensioni (fig. 14.2).

B11. Cornice a fascia piatta con decorazione a kymation lesbico; ne restano due frammenti: $\mathrm{cm} .3$ e 4 di lungh; largh. cm.1; spess. cm. 0,2 (fig. 14.3).

B12. Frammento di grappolo, del quale sono riconoscibili tre frammenti non combacianti: h. del frammento maggiore $\mathrm{cm} .2,1$; largh. cm. 1,2; spess. cm. 0,5 (fig. 15).

B13. Cornice a "scorza di palma". Ne restano 5 frammenti dei quali 4 combacianti: la lungh. di quelli ricostruiti è di $\mathrm{cm}$. 7 ; lo spessore medio è di cm. 0,4; largh. cm. 0,7 (fig. 14.4).

B2 - B9. Figura principale:

- Volto di incerta classificazione (dea o Dioniso), finito orizzontalmente a metà fronte, nel punto in cui doveva essere un altro elemento (una coroncina o forse un'acconciatura); folti boccoli incorniciano il viso; tratti somatici ben definiti (fig. 16).

- Ala sinistra appartenente alla figura principale: h. cm. 3,5; largh. cm.1,5; spess. cm. 0,4.

- Ala destra appartenente alla figura principale: h. cm. 1,3; largh. cm.1; spess. cm. 0,4.

- Frammento di ventre nudo a parte di panneggio appartenente alla figura principale: h. cm. 0,9 ; largh. cm. 3,2; spess. cm. 0,4.

- Frammento di gamba, costituito da polpaccio, ginocchio e parte della coscia della gamba sinistra, appartenente alla figura principale: h. cm.3, largh. cm.1,2; spess. cm. 4 .

- Mano sinistra stringente il panneggio, appartenente alla figura principale: h. cm. 1,6; largh. cm. 2; spess. cm. 0,6 (fig. 17).

- Frammento della spalla e dell'avambraccio sinistro con panneggio appartenente alla figura principale: h. cm. 2,5; largh. cm. 1,5; spess. cm. 0,5.

\section{ELEMENTI PERTINENTI AL FULCRUM}

- Frammento di lamina piatta e decorata, di rivestimento, con cornice a fascia incorporata: $\mathrm{h} . \mathrm{cm}$. 1; largh. cm. 1,7; spess. cm. 0,3.

- Tre frammenti variante del precedente.

- Variante del precedente: presenta una leggera curvatura verso l'interno: h. cm. 2; largh. cm. 3; spess. cm. 0,3.

- Medaglione decorato di cui restano solo 8 frammenti: diam. cm. 10; spess. cm. 0,4. Non si distingue il tipo decorativo.

\section{ELEMENTI DI INCERTA COLLOCAZIONE}

- Gallo acefalo: h. cm. 2,4; largh. cm. 2,7; spess. cm. 0,5 (fig. 18).

- Un amorino citaredo in corsa verso destra: $h$. cm. 3; largh. cm. 2,5; spess. cm. 0,8.

- Due amorini citaredi in corsa verso sinistra. Mancano della gamba destra: h.cm. 2,4; largh. cm. 1,8 ; spess. cm. 0,5 (fig. 19).

- Anello cilindrico ricavato da un unico osso tubolare, costituente il supporto delle figure ed innestato direttamente sulla verga in ferro di sostegno delle gambe. Incompleto: h. cm. 5,6; spess. cm. 0,6; diam. max. cm. 2,5; diam. min. cm. 1,4.

- Elemento ad incastro con funzione di supporto per le figure, a sezione semicilindrica, ricavato da osso tubolare: h. cm. 4; largh. cm. 1,5; spess. cm. 0,3 .

- Frammento di testa, viso e ala di amorino volto verso sinistra: h. $\mathrm{cm} .2$; largh. $\mathrm{cm} .1,7$; spess. $\mathrm{cm}$. 0,4 .

- Frammento di mano con cornucopia. h. $\mathrm{cm}$. 2,6; largh. cm. 1,8; spess. cm. 0,4 (fig. 20)

- Frammento di gamba sinistra: h. cm. 3; largh. cm. 1,8 ; spess. 0,4 .

- Frammento di gamba sinistra con piede, probabilmente di amorino: h. cm. 1,7; largh. cm. 0,7; spess. cm. 0,3.

- Variante del precedente, gamba sinistra con ginocchio piegato: $\mathrm{h}$. cm. 1,6; largh. cm. 1; spess. cm. 0,3 .

- Frammento di ala con penne larghe e sottilmente incise: h. cm. 1,3; largh. cm. 1,7; spess. cm. 0,3 ( fig. 21).

- Frammento di ala o di panneggio: h. cm. 2; largh. cm. 1,5; spess. cm. 0,5.

\section{FERRO}

- Chiodi con capocchia larga e bombata: h. cm. 1,5; diam. cm. 1,1; spess. della capocchia cm. 0,6.

- Chiodi a sezione quadrangolare con capocchia arrotondata: h. cm. 6; spessore dell'asta cm. 0,6; diam. cm. 2.

- Chiodi a sezione triangolare con capocchia quadrata: h. cm. 4; spess. dell'asta cm. 0,3; largh. della capocchia $\mathrm{cm}$. 1,5. Tutti i chiodi risultano molto deformati dalla ruggine.

\section{BRONZO}

- Piccoli chiodi con capocchia circolare ombelicata: h. cm. 0,3; diam. cm. 0,4. 


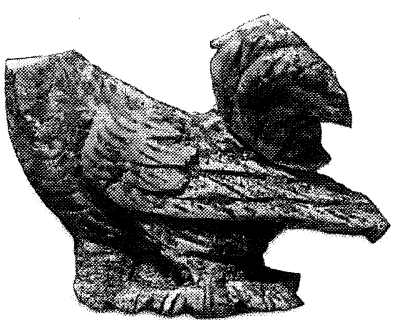

18

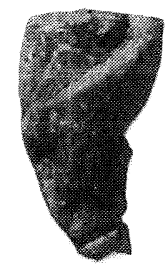

20

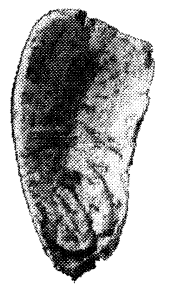

21
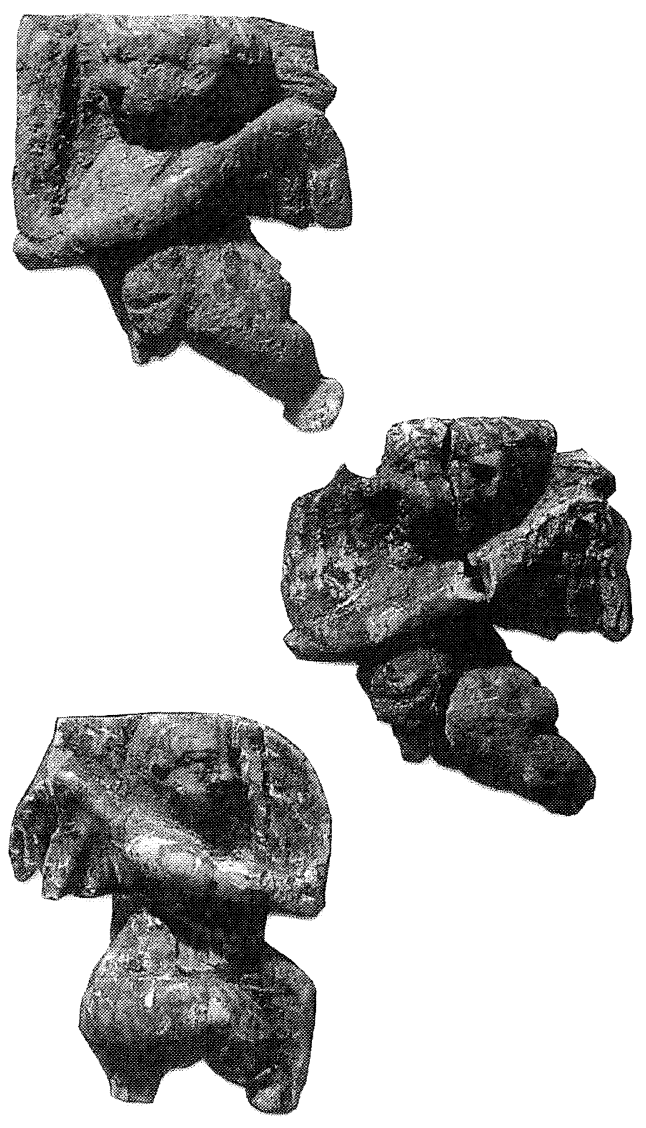

19

Figg. 18 a 21.-18: gallo acefalo; 19: amorini citaderi di incerta collocazione; 20: mano con cornucopia; 21: frammento di ala.

\section{RICOSTRUZIONE (figg. 22 e 23)}

La ricostruzione è stata basata su parametri tecnici, sul confronto dei diametri, interni ed esterni, degli anelli posti intorno alla verga ${ }^{6}$, e sull'analisi degli incassi dei diversi elementi. In particolare si è rivelata insostituibile la ricostruzione effettuata dal Nicholls di un letto di provenienza ignota, che presenta notevoli analogie con il letto di La Cona ${ }^{7}$.

${ }^{6}$ La cospicua presenza di ruggine sulla maggior parte dei fr. ossei rimasti suggerisce l'impiego di verghe in ferro per le gambe. Tale ipotesi troverebbe conferma anche nelle misure relative ai diametri degli anelli posti intorno alla verga stessa, piuttosto ridotte. L'assenza delle verghe in ferro potrebbe essere dovuta alla pratica dell'ossilegium o solo per motivi pratici.

${ }^{7}$ Nicholls 1979, passim.

\section{GAMBA}

L'anima della gamba ${ }^{8}$ doveva essere costituita da una verga lunga intorno ai $60 \mathrm{~cm}$. ed avente il diametro di $1,4 \mathrm{~cm}$., come appare confermato dal diametro interno di tutti gli anelli esaminati; una rondella, con ogni probabilità in ferro, posta all'estremità inferiore dell'asta serviva a bloccare gli anelli, che venivano infilati dall'alto in successione.

${ }^{8}$ Cfr. Letta 1984, p. 77. La scelta della verga in ferro o in legno è probabilmente subordinata ad esigenze strutturali ed economiche, come del resto anche l'altezza delle gambe era legata a criteri di stabilità; il Letta fa notare inoltre come sia una particolarità dei letti antichi, sia in bronzo che in avorio e in osso, l'altezza leggermente maggiore delle gambe del lato destinato alla testa rispetto al lato dei piedi. 


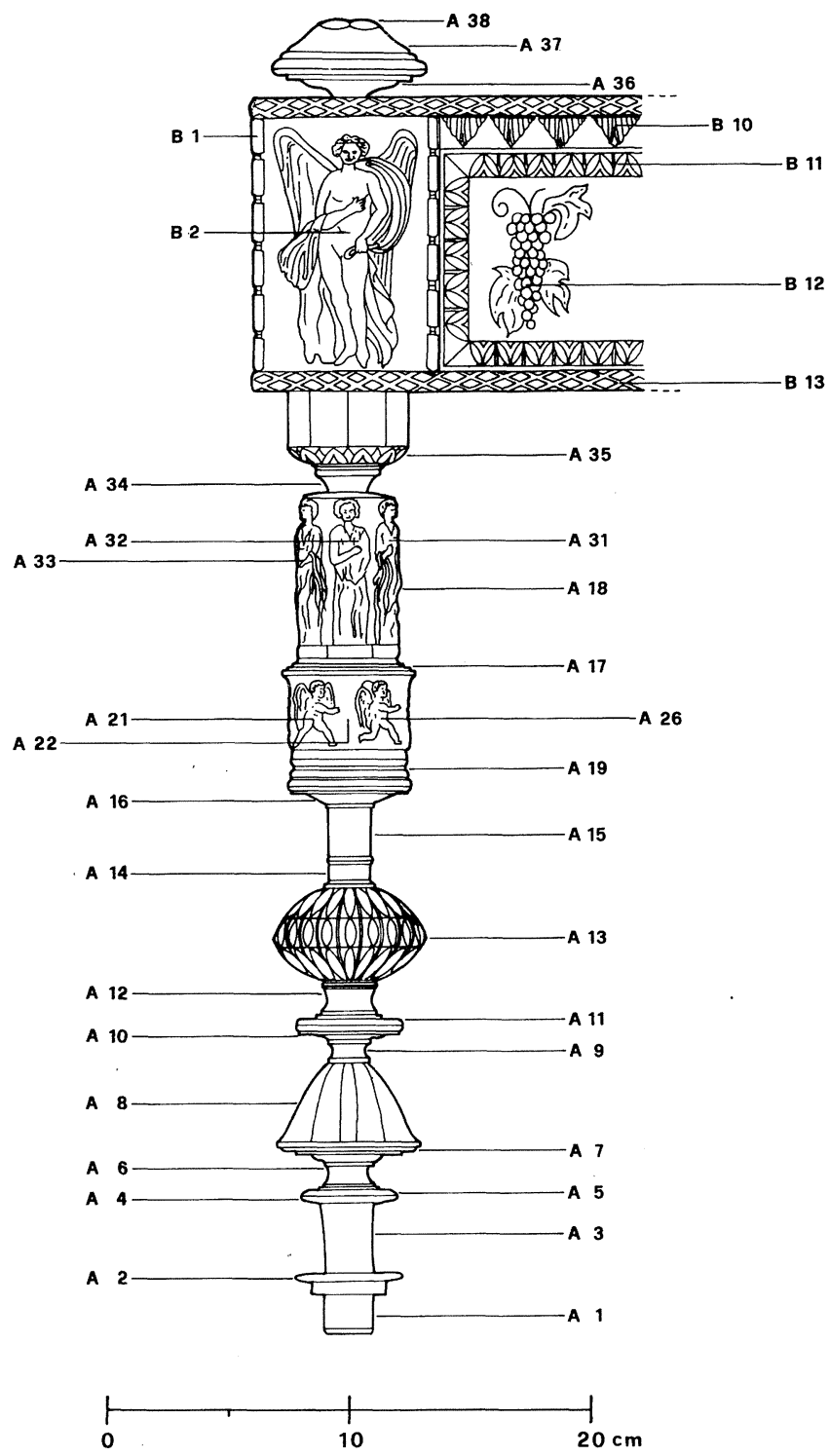

Fig. 22.

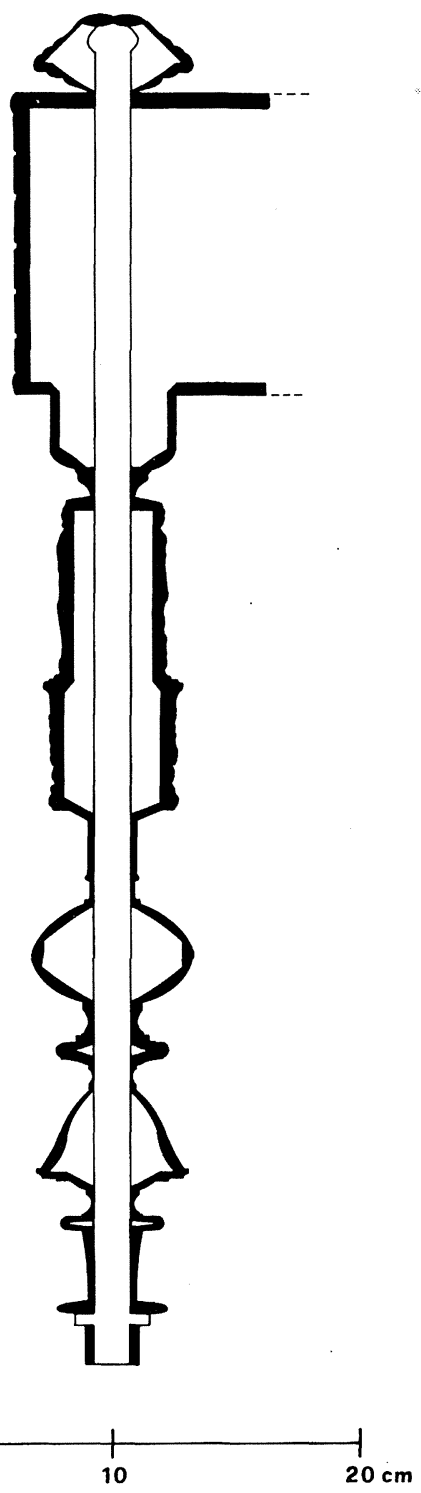

Fig. 23.
Procedendo dal basso verso l'alto si ottiene:

1. Puntale affusolato A1, ricavato da un unico osso ${ }^{9}$ tubolare tornito e provvisto di incassi interni ai bordi; su questo si innesta una rondella in ferro sulla quale si appoggia un anello tornito a sezione piena $\mathrm{A} 2$; un anello tornito a profilo leggermente concavo con incasso interno ai bordi $\mathrm{A} 4 \mathrm{e}$ un anello tornito a profilo leggermente convesso con dente

${ }^{9}$ Le ossa usate in genere appartenevano a bovini o a cavalli poiché di larga superficie e dunque più facilmente utilizzabili per trarne elementi decorativi costituiti da un unico pezzo. che crea una bordatura orizzontale A5 sono opposti e combacianti tra loro e costituiscono il punto di giunzione di A1 con il successivo A3 elemento tubolare avente le stesse caratteristiche di A1.

2. Campana liscia composta da più lamelle combacianti tra loro avente profilo stretto e allungato A8; essa è chiusa in basso da un anello a profilo leggermente convesso A7; l'insieme è compreso e definito dalle strozzature A6 e A9, elementi ricavati da un unico osso tubolare e torniti; l'anello tornito e profilato A10 e l'anello tornito con profilo comprendente una metà concava ed una convessa $\mathrm{A} 11$, opposti $\mathrm{e}$ combacianti fra loro, si configurano come una "pau- 
sa" nella scansione di elementi maggiori come la campana e il successivo.

3. Toro costituito da due anelli ed una fascia ricavata da più lamelle decorate con un motivo di foglie d'acqua stilizzate A13; la fascia centrale separa i due anelli opposti tra loro determinando la forma globulare dell'insieme; come accade per la campana due strozzature A12 e A14, analoghe nella morfologia a quelle già viste, concludono $\mathrm{A} 13$ in basso e in alto, mentre l'elemento tubolare tornito A15 anch'esso con incassi interni ai bordi, conferisce snellezza all'insieme, costituendo lo stacco ideale fra gli elementi sopra descritti e il cilindro figurato.

4. Cilindro figurato articolato su due registri di diversa dimensione, A18 e A22; un elemento ricavato da un osso tubolare non tornito costituisce il punto di raccordo tra i due registri. Quello inferiore A22 più piccolo, largo e schiacciato è caratterizzato da una fascia anulare modanata composta da più parti A19 e poggiante sull'anello a profilo convesso A16, che costituisce il campo decorativo sul quale amorini citaredi in corsa A21-A26 si articolano in successione. Sul registro superiore, più stretto e slanciato del primo, si allineano su base modanata alcune figure panneggiate A31-A33 ${ }^{10}$. A18 secondo la ricostruzione ipotizzata viene ad avere un'altezza di $\mathrm{cm}$. 14 ed è concluso in alto da una strozzatura A34 simile alle precedenti.

5. Un capitello liscio era probabilmente agganciato direttamente al telaio, nella sua parte superiore mentre quella inferiore è chiusa da un giro formato da una sottile lamina decorata da ovoli (A35).

6. L'estremità superiore della verga, sopra il telaio, era rivestita dagli anelli A36 e A37, combacianti fra loro, ai quali si sovrapponeva una borchia lenticolare (A38) che, secondo Letta ${ }^{11}$, aveva nei letti di bronzo la funzione di supportare il fulcrum, qui invece utilizzata a scopo decorativo.

\section{TELAIO}

Per quanto riguarda il telaio del letto la possibile collocazione della figura alata B2 suggerisce un'altezza di questo di circa $12,5 \mathrm{~cm}$, misura con-

${ }^{10}$ Riguardo a questo tipo di cilindro figurato su due registri si veda Talamo $1987-88$, pp. $2-43$ e p. 80 ; il registro inferiore con gli amorini sembra inoltre potersi ricollegare alla descrizione fornita dal Letta 1984, p. 86 del cilindro figurato di tipo II, raffigurante amorini in corsa intorno ad un trofeo d'armi. Questo tipo di cilindro ha dimensioni minori rispetto a quello figurato principale ed è associato ad esso.

11 Letta 1984 , p. 72 e 77. forme agli esempi documentati ${ }^{12}$. Cornici a bastoncello semicilindriche del tipo ad astragali B1, inquadravano la figura alata che rientra in una tipologia ampiamente documentata ${ }^{13}$. Il volto B3 finito orizzontalmente a metà fronte nel punto in cui attaccava probabilmente un altro frammento ora mancante, presenta la definizione accurata dei tratti somatici, con folti boccoli che lo incorniciano; due grandi ali superano in altezza la testa della figura e scendono fino ai piedi, nascoste a tratti dall' ampio panneggio. Sottili incisioni esaltano la resa dei particolari, quali le piume delle ali e le pieghe del panneggio. Realistici e curati nella morfologia e nelle proporzioni sono gli altri elementi, come il frammento di ventre nudo, il polpaccio, parte del ginocchio e della coscia della figura, un frammento comprendente parte della spalla e dell'avambraccio sinistro e infine la mano sinistra della figura che con eleganza stringe la parte terminale del morbido panneggio.

L' estremità superiore del telaio, lungo la sua fascia centrale, doveva essere delimitata dall'elemento ricavato da un osso tubolare inciso e lavorato a foglia d'acanto a lingua ricurva B10, del quale restano numerosi esemplari posti in successione; la parte inferiore del telaio era ornata da cornici dette a scorza di palma per la loro caratteristica lavorazione B13. La fascia centrale doveva essere costituita da pannelli decorati con motivi vegetali, come sembra indicare con buona probabilità l'elemento B12, identificato come grappolo d'uva; i pannelli erano inquadrati da una sottilissima fascia decorata con kymation lesbico B11.

\section{FULCRUM}

Dei fulcra, come si è detto, non rimane praticamente nulla tranne parte della lamina decorata di rivestimento con cornice incorporata ${ }^{14}$ che seguiva l'andamento sinuoso del fulcrum. Alcuni frammenti relativi ad un disco con decorazione, tornito e di largo diametro, suggeriscono la presenza di un medaglione. La mancanza di un numero sufficiente di frammenti e la loro eccessiva deformazione non ha consentito un'ipotesi di ricostruzione grafica.

12 Circa le dimensioni canoniche dei letti si veda Talamo 1987-88, p. 54.

13 La figura alata è descritta nelle sue varianti da Letta 1984 , p. 86 che la colloca nel cilindro di tipo I. La Talamo 1987-88 suggerisce minore rigidità nelle attribuzioni strutturali dei tipi figurati.

14 L'andamento sinuoso della lamina rinvenuta in più frm. ha suggerito l'appartenenza di questa alla decorazione del fulcrum. Non si tratta infatti di una deformazione dovuta al calore della combustione, dato che i frammenti rimasti combaciano perfettamente e aderiscono in una composizione finita. 


\section{CONFRONTI STILISTICI ED INQUADRA- MENTO CRONOLOGICO Per Silvia Barbetta}

La ricostruzione grafica proposta è stata basata, come abbiamo visto, su parametri prevalentemente tecnici.

Considerando il profilo propriamente artistico si può notare che mentre da un lato alcuni degli elementi ornamentali assolvono ad un compito oltre che decorativo anche funzionale alla struttura del letto -e sono dunque ricorrenti- dall'altro le raffigurazioni principali, spesso ispirate ai miti o alle rappresentazioni allegoriche, assumono caratteri propri dipendendo dal gusto dell' artigiano o del committente e senza dubbio dalla moda del momento.

E' possibile richiamare numerosi confronti tipologici per quanto riguarda gli elementi morfologici qui individuati. L'elemento a campana ed il "toro" ${ }^{15}$, la serie variamente diametrata degli anel$\mathrm{li}^{16}$, alternata agli elementi tubolari ed alle strozzature ${ }^{17}$, riconducono, sia pure con qualche inevitabile variante, ad uno schema univoco, quello della gamba "tornita" ${ }^{18}$. Anche altri elementi, analizzati singolarmente, sono consueti e ricorrenti nel repertorio della maggior parte dei ritrovamenti di questo tipo: dai più antichi letti di Ostia a quelli più recenti di Vindonissa si ritrovano, ad esempio, le cornici rettilinee, riferibili al telaio e definite a "scorza di palma" per le loro particolari incisioni a losanghe, quelle cosiddette a "foglia d'acanto ricurva" e la decorazione a "kymation lesbico" (fig. 14) ${ }^{19}$

All'interno della produzione di letti funebri, il Letta ha riconosciuto tre tipologie: una costituita da letti con decorazione in avorio (la cui diffusione in area italica è piuttosto scarsa e limitata) e altre due distinte a seconda della presunta derivazione da modelli di letti con decorazione eburnea o bronzea ${ }^{20}$.

15 V. supra la ricostruzione a p. 302 , nn. 2 e 3 .

16 A 2, A 4, A 5, A 16, A 19.

17 A 1, A 3, A 6, A 12, A 15, A 34.

18 Cfr. Letta 1984, p. 98 n. 45; p. 105 n. 109; p. 106 n. 121. A proposito delle gambe "tornite" cfr. C. L. Ransom, Studies in ancient furniture. Couches and beds of the Greek, Etruscans and Romans, Chicago 1905, p. 32 e G. M.A. Richter, The Furniture of the Greeks, Etruscans and Romans, London 1966, pp. 105 ss.

19 B 13, B 10, B 11. Si ritrovano infatti nei letti di Norcia (cfr. Pasqui 1890, pp. 234-244; Letta 1984 p. 104 nn. 97-98), Ostia, S. Vittore di Cingoli, Fagnano Alto, Ancona, Corfinium, Pianella, Maltignano di Cascia, Aquino, Cremona ed infine a Roma (cfr. Letta 1984, p. 97 nn. 34-36; p. 101 n. 76 e 79 ; p. 109 nn. $81-83$; p. 103 n. 85 ; p. 105 nn. $102-103$ e 109; p. 106 nn. 114-452; Talamo, 1987-88, p. 59, fig. 69).

20 Letta 1984, pp. 94-111. Il catalogo comprende anche una serie di letti di incerta classificazione. Da segnalare inoltre frammenti di decorazione in osso da S. Pietro in Casale
Esiste un'effettiva difficoltà a ricondurre gli elementi del letto di Teramo alle caratteristiche di una sola di queste categorie e ciò rivela gli aspetti di una produzione artigianale, la quale, riflettendo gusti e preparazione tecnica diversi per ogni maestranza, non è agevolmente classificabile ${ }^{21}$.

Forse più facile è l'attribuzione ad una delle due distinte correnti artistiche individuate dalla Talamo: una estremamente raffinata e direttamente derivata dall'arte ellenistica, propria di letti in avorio ed in osso, ed un'altra che, invece, utilizza elementi più massicci e rozzamente lavorati, caratteristica solo dei letti in osso e tipica dell'Italia centrale.

Nel nostro caso, non solo alcuni degli elementi figurati risultano eseguiti con l'attenzione e la perizia propri di lavori di alto livello qualitativo, come il corpo del gallo (fig. 18), la testina ricciuta (fig. 16) ed alcune figurine di putti (figg. 11 e 19), ma anche la resa slanciata di tutta la gamba, consentita dall'inserimento di strozzature e di elementi tubolari ed il rispetto, nella sintassi decorativa, di "una complessa composizione figurativa composta da una serie di personaggi su una base modanata (corteo di Amorini alati)", considerata indicativa di una maggiore raffinatezza del prodotto ${ }^{22}$.

Tutto ciò suggerisce per il letto di Teramo l'appartenenza alla corrente più elegante, di cui l'esempio più noto è senza dubbio il letto di Cambridge ${ }^{23}$; la presenza del galletto, inoltre, unico elemento diverso e raramente presente nei letti conosciuti -e che dunque non trova alcun confronto utile ${ }^{24}-$ è ulteriormente indicativa della ricercatezza posta nell'esecuzione.

Troppo poco si è conservato del rivestimento per consentire una corretta ricostruzione del tema iconografico; a livello indiziario, i piccoli amorini citaredi i quali, sovrastati da una teoria di ninfe, si affiancano alla grande figura alata, cinta da un am-

(BO) e da Cortemaggiore (PC) per i quali v. rispettivamente G. A. Mansuelli, Il monumento funerario di Maccaretolo e il problema dei sepolcri a cuspide in Italia, in Archeologia Classica IV, 1952, pp. 60-61 e P. Saronio, Cortemaggiore (Piacenza). Area SIPEM. Necropoli romana, in Bollettino di Archeologia 5/6, 1990, p. 124.

21 Sul problema cfr. anche Talamo 1987-88, p.78

22 Talamo $1987-88$, p. 76

23 Talamo $1987-88$, p. 76 . In effetti sembrano molto distanti dai tratti duri e provinciali delle decorazioni dei letti, ad esempio, di Amplero. Cfr. M. Faita, Due letti funerari con rivestimento in osso da Aielli (AQ), in SCO XXXIX, 1989, pp. 281-309 e il catalogo della mostra: Amplero. Archeologia e storia di un centro italico romano: 20 anni di ricerche, 1989 , pp. 34-38. Per una rivalutazione del prodotto italico cfr. Ortalli 1991, p. 118.

${ }_{24}$ L'unico caso in cui si ha notizia di un gallo è nella protome di un fulcrum proveniente da Colonia (Letta 1984, p. $86 ;$ p. 87 e p. 111 n. 182 ) 
pio panneggio, trovano echi diretti nel tema della Venus Pudica del letto di Cambridge; tuttavia la contestuale presenza di un frammento, interpretabile come grappolo d'uva (fig. 15), il frammento che raffigura una mano che sorregge una cornucopia (fig. 20) ${ }^{25}$, la relativa quantità di amorini e di frammenti di ali, sembrano ricondurre in maniera univoca a raffigurazioni del tiaso bacchico, uno dei soggetti più ricorrenti nella decorazione dei letti funebri ${ }^{26}$. Sembra avvalorare quest'ultima ipotesi la presenza del galletto, indicativo di una simbologia funeraria ben nota ${ }^{27}$ : oltre ad essere molto spesso connesso al grappolo d'uva, il significato di resurrezione che gli viene attribuito può ricondurre a Dioniso, quando lo stesso dio è simbolo di morte e resurrezione ${ }^{28}$. Così, anche la stretta connessione

${ }^{25}$ Confronti precisi in $\mathrm{H}$. Brunn, Lavori intagliati in osso, in AnnInst XXXIV, 1862, pp. 284-287, tav. P; cfr. anche W. Frohener, Collection Hoffmann, Paris 1888, II, tav. XLI, dove però la mano sorregge un tirso.

26 L'ampia diffusione di questo tema nelle raffigurazioni note è testimoniata dall' alta percentuale di presenze della figura di Dioniso (esemplari da Pompei, Vindonissa, da un letto conservato a New York e da altri di provenienza ignota: Letta, p. 95 n. 3 ; p. 96 n. 23; p. 98 n. 44; p. 99 nn. 47 e 51; p. 106 n. 116); di menadi e di personaggi bacchici (provenienti da Ancona, Castelvecchio Subequo, Pianella, S. Maria degli Angeli, Orvieto, Cremona, Palençia e più genericamente dall' Abruzzo: Letta 1984, p. 101 n. 76; p. 102 n. 84; p. 103 n. 85 ; p. 104 nn. 93-94; p. 105 nn. 105-106; p. 106 n. 121; p. 107 nn. 123-124); anche da Aosta proviene un letto "ornato di un fregio probabilmente a carattere dionisiaco": cfr. R. Mollo Mezzena, Augusta Praetoria. Aggiornamento sulle conoscenze archeologiche della città e del suo territorio, in $R S L$ XLI-XLII, 1975-1976, 1982, pp. 147-257, p. 208. Satiri e sileni sono anche spesso presenti come culmini dei fulcra come ad Atene, Monte Sannace, Pompei, Ancona, Canusium, Mimerki, Phanagoria e Tiritaki, in un letto conservato a Londra ed in uno a Parigi ed in altri di provenienza ignota (Letta 1984, nn. dal 4 al 10 e p. 99 nn. dal 53 al 59).

27 V. Macchioro, Il simbolismo nelle figurazioni sepolcrali romane, in MemNap I, 1911, pp. 96-102; sul significato ctonio del sacrificio del gallo v. R. Turcan, Les sarcophages romains a représentation dionysiaques, in BEFRA 210,1966, p. $496 \mathrm{ss}$. Sul potenziale valore funerario di tutte le iconografie bacchiche cfr. Letta 1984, p. 91 e n. 48. La pertinenza all'ambito funerario della presenza di Dioniso, anche solo simboleggiata dal grappolo d'uva e dal galletto è evidenziata anche da P. Zancani Montuoro, Note sui soggetti delle tabelle di Locri, in AttiMemSocMagna Grecia, n.s. I, 1954, pp. 71-106: i pinakes del santuario di Persefone a Locri, della prima metà del V sec.a.C., raffigurano Persefone, con o senza Hades, alla quale Hermes porge in dono un gallo; a volte compaiono sia il gallo che i grappoli d'uva simboleggianti Dioniso; altrove Persefone stessa ha in mano un gallo mentre Dioniso è al suo cospetto.

${ }^{28} \mathrm{R}$. Stuveras, Le putto dans l'art romain, in Latomus XCIX, 1969, pp. 180-196. Sul rapporto tra amorini e galli in ambito funerario cfr. anche Turcan, cit. (n. 27), p. 560 e p. 614 per i combattimenti di galli sui sarcofagi. Una raffigurazione di Eroti con galli da combattimento è su un cammeo conservato al Museo Nazionale di Napoli: cfr. A. Giuliano, $I$ Cammei della Collezione Medicea nel Museo Archeologico di Firenze, Roma 1989, p. 42. D'altra parte, in diverso rapporto e quindi con valenza iconografica diversa, amorini tra putti e Dioniso, laddove quest'ultimo assume un significato ctonio, rivela il carattere funerario che gli stessi possono ricoprire.

Sembra, in definitiva, plausibile riconoscere nella decorazione del letto di Teramo - La Cona un'iconografia bacchica, interpretata e giustificata dal Letta per l'ambito funerario come "simbolo di apoteosi mistica".

Anche gli elementi relativi ai fulcra sono estremamente esigui per poter proporre la ricostruzione iconografica. Morfologicamente non dovevano differire dal tipo sinusoidale, canonico per questa classe di letti e articolato in tre sezioni: la prima costituita da medaglioni che rappresentavano maschere gorgoniche, teste maschili -con elmo o senza- o figure femminili; la seconda da campi centrali variamente decorati ${ }^{29}$; la terza da culmini consistenti principalmente in teste di animali, poichè facilmente adattabili alla morfologia terminale del fulcrum ${ }^{30}$. Così ritroviamo di preferenza teste di uccelli, anche acquatici, come cigni ed oche ${ }^{31}$, teste di cavalli o di muli ${ }^{32}$, protomi di linci o di leoni ${ }^{33}$ e teste di satiri o di sileni ${ }^{34}$; particolari un pellicano di provenienza ignota, delfini da Maltignano di Cascia, una testa di cobra da Colonia e probabilmente sfingi nel letto di S. Lorenzo in Strada ${ }^{35}$.

Non sembra possibile stabilire altre associazioni tra la decorazione del letto ed il tipo di fulcrum se non nel caso delle protomi a testa di mulo e quelle con satiri e sileni, che possono essere facilmente ri-

sono associati ai gruppi con Amore e Psiche, come a Bitia ed a Colonia (Letta 1984, p. 97 n. 37; p. 98 n. 39), ed a quelli della Venus Pudica, nel letto di Cambridge. Eroti intenti a svolgere qualche attività di gruppo, come la vendemmia o scene di caccia o di lotta, dovevano ornare il letto di S. Lorenzo in Strada, tra Rimini e Riccione, datato nella seconda metà del I sec. d.C.: cfr. Ortallu 1991, pp. 106-107.

${ }^{29}$ Letta, 1984 , p. 97 n. 36 ; p. 99 nn. 48-49; p. 98 nn. 4041 e nn. 43 e 46 ; p. 101 nn. $78-79$, n. 80 , nn. 81-83; p. 103 nn. 87 e 92 , pp. 86-87.

${ }^{30}$ Per l'evoluzione tipologica dei fulcra cfr. Letta 1984, pp. 82-84 e 89-90. Per una classificazione dei tipi di fulcra v. da ultima S. Faust, Fulcra. Figürlichter und ornamentaler Schmuck an antiken Betten, in $M D A I(R), 30,1989$, pp. 155156.

31 A Pompei, Fagnano Alto, Ancona, Penne, Praeneste, nel letto di New York, a Roma ed in un letto di provenienza ignota (Letta, cit., p. 97 n. 36; p. 99 nn. 49-50; p. 100 nn. 63. $65,68,69$ e 71); Vaglieri in $N S c$ 1912, p. 98 accenna, tra l'altro, alla presenza di un cigno nella tomba di Ostia.

32 A Corfinium, Norcia e Cremona (Letta 1984, p. $102 \mathrm{nn}$. $81-83$; p. 104 n. 99 ; p. 106 n. 121 ).

${ }^{33}$ Ancora da Ancona, Penne, Aielli, Norcia, Orvieto (Letta 1984, p. 101 nn. 77-78; p. 103 n. 86; p. 104 n. 97; p. 105 nn. 105-106).

${ }_{34}$ Da Ancona, Pompei, Canusium (Letta 1984, p. $100 \mathrm{nn}$. 56-57 e 59).

35 Letta,1984, p. 99 n. 48; p. 105 nn. 102-103; p. 107 n. 126. Sui motivi più ricorrenti nei fulcra $\mathrm{cfr}$. anche Richter 1966, pp. 107-108. 
condotti all'iconografia bacchica, o in quello delle protomi a testa di cigno o di oca, che sembrano essere pertinenti alle raffigurazioni di Amore e Psiche o di Afrodite; la scelta, guidata dalla morfologia del fulcrum, deve aver avuto anche sicure motivazioni allegoriche ${ }^{36}$ :

L'introduzione in Italia di tali mobili, derivanti dai modelli ellenistici in avorio o altro materiale prezioso ${ }^{37}$, sembra attestata fin dalla seconda metà del III secolo a.C., come indicano gli esemplari rinvenuti a Monte Sannace e Praeneste ${ }^{38}$ (fig. 29). Confronti puntuali degli ornati del letto di Teramo-La Cona restringono però l'arco cronologico: infatti l'esame dei repertori evidenzia la diffusione delle cornici decorate con "kymation lesbico" e a "scorza di palma" dalla fine del II secolo a.C.; quest'ultima tuttavia risulta assente in diversi contesti ed appare meno frequentemente del kymation. Le foglie d'acanto incurvate a lingua, sembrano invece diffondersi maggiormente in età augustea e giulio-claudia. Tra le scene figurate, decorazioni con amorini si ritrovano ad Ostia già nella seconda metà del II secolo a.C. ${ }^{39}$, come anche motivi simili appaiono nei letti di Norcia ed Ancona, che rientrano nell'ambito dello stesso secolo. Più circoscritta cronologicamente è l'apparizione dei letti in osso in Abruzzo, datata tra la metà del I secolo a.C. e gli inizi del successivo, arco di tempo che corrisponde anche al periodo della loro maggiore diffusione in territorio italico (fig. 31) ${ }^{40}$.

Nel caso del letto di Teramo, un fattore di notevole importanza ai fini di una determinazione cronologica è rappresentato dal rinvenimento dei frammenti ossei in un contesto archeologico relativamente integro ${ }^{41} \mathrm{e}$ dal quale si ha una conferma alla cronologia proposta in base ai riscontri stilistici. Il materiale associato colloca, infatti, la tomba nella prima metà del I secolo d.C.: le lucerne a vo-

${ }^{36}$ Sui fulcra e sulle eventuali associazioni con la decorazione cfr. Letta 1984, p. 83

37 Per i differenti materiali utilizzati cfr. Richter 1966, p. 122 sg. e Ransom 1905, p. 54. Sulla tecnica cfr. da ultimo A. Mac Gregor, Bone antler Ivory and Horn, London - Sidney 1985.

38 Letta 1984 , p. 95 n. 5 ; p. 100 n. 67

39 D. Vaglieri in NSc 1912, p. 98 fig. 10. Gli amorini riprodotti sono del tutto simili ai nostri, tranne che per la posizione delle braccia. Il Vaglieri non parla di letti funebri, ma più in generale di mobili o urne funerarie, come anche $\mathrm{N}$. Brizio (NSc 1902, p. 580) considera gli ossi lavorati di Ancona pertinenti ad urne o cofanetti "cari al defunto". I disegni di alcuni putti della tomba di Ostia si ritrovano in S. Reinach, Répertoire de la Statuarie grecque et romaine, V, 1 , Paris 1924, p. 193 nn. 6-8.

${ }^{40}$ Letta 1984, pp. 92-93: secondo l'autore l'uso dei letti in osso per il rito funebre si diffonde nelle province a partire dal i secolo d.C.

41 Per le condizioni della tomba al momento del rinvenimento cfr. supra pp. 295-6; Ortalli 1991, p. 102 e 104 s.

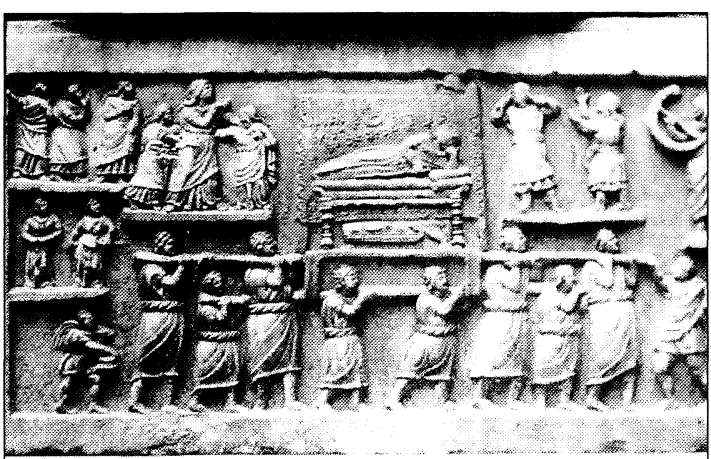

Fig. 24.-Bassorilievo con processione funebre de Amiternum

lute con spalla interrotta e disco decorato a fiore ${ }^{42}$, i balsamari in vetro ${ }^{43}$, i frammenti di ceramica sigillata e altre forme vascolari (oinochoai in depurata), riconducono allo stesso periodo. Presentano inoltre confronti più puntuali con il nostro le decorazione a kymation lesbico del letto di Fagnano Alto e dei frammenti di S. Vittore di Cingoli, datati rispettivamente alla fine del I secolo a.C. ed al primo quarto del I secolo d. C. ${ }^{44} \mathrm{e}$, fuori del circoscritto ambito territoriale, i frammenti di Vindonissa, tra cui amorini e cornici a "scorza di palma", del secondo quarto del I secolo d.C. ${ }^{45}$.

In conclusione, il letto teramano rientra nella scia di una antica tradizione della lavorazione del mobilio che, a prescindere dallo specifico uso funerario o domestico, amava "tornire" le gambe di letti, sedili e seggi. Il gusto per gli arredi con le gambe "tornite" si ritrova infatti documentato in altre espressioni artistiche come raffigurazioni vascolari, sarcofagi (fig. 24) ${ }^{46}$ e soprattutto affreschi (figg.

${ }^{42}$ S. Loeschcke, Lampen aus Vindonissa, Zurich 1919, tipo I A, inizi I sec. d. C.

${ }^{43}$ K. Goethert-Polaschek, Katalog der römischen Gläsers Rheinischen Landesmuseum Trier, Mainz a. Rhein 1977, tav. II.

${ }^{44}$ Letta 1984, p. 97, n. 36; L. Mercando, S. Vittore di Cingoli, in NSc 1974, p. 123.

${ }_{45}$ Per la documentazione del materiale di Vindonissa, riferibile a letti in osso, di cui manca purtroppo la ricostruzione v. Th. Eckinger, Knochenschnitzerein aus Gräbern von Vindonissa, in Anzeiger fur schweitzerische Altertumskunde, XXXI, 1929, tavv. XXXVIII-XXXIX; cfr. anche Talamo, 1987-88, p. 93 fig. 117 e p. 95 fig. 118.

${ }^{46}$ In particolare per l'ambito territoriale interessato da questo studio è significativo il letto della processione funebre raffigurata su un sarcofago proveniente da Amiternum, della prima metà del i secolo d.C. Cfr. H. Stuart Jones, $A \mathrm{Ca}$ talogue of the Ancient Sculpture preserved in the Municipal Collection of Roma. The Sculpture of the Palazzo dei Conservatori, Oxford 1926, p. 75 n. 11; R. Bianchi Bandinelli, Roma. L'arte romana al centro del potere, Milano 1981, p. 59 ; L. Franchi dell'Orto, Rilievo con pompa funebre e rilievo con gladiatori al museo dell'Aquila, in Studi Miscellanei $10,1963-1964$, pp. 39-55, in particolare p. 25. 
$25,26,27$ e 28$)^{47}$, ma anche su dischi figurati di lucerne e nella glittica ${ }^{48}$.

Accanto ai modelli con decorazione in avorio, si sviluppò un artigianato che, con la lavorazione dell'osso ad imitazione del materiale più prezioso, consentì una diffusione capillare di questa nuova moda. Suggestiva, a questo proposito, l'osservazione del Letta sui tentativi di originalità espressiva, riscontrabili nella produzione dei letti in osso, che tenderebbero così ad affrancarsi dai precedenti modelli eburnei e bronzei, nella ricerca di una tradizione propria ${ }^{49}$. La persistenza dei modelli, che permette confronti anche con letti più antichi, trova spiegazione, specie in questo ambito provinciale, nei tentativi di imitare e fare proprio il lusso di un'arte preziosa, nata nelle corti ellenistiche e prontamente recepita dal mondo romano ${ }^{50}$. Ciò si riscontra soprattutto in epoca augustea, quando l'arte "come il principato di Augusto si regge sopra una finzione: quella di essere la continuità della tradizione dei regni ellenistici..." ${ }^{51}$. E' dunque plausibile, come giustamente suggerito dalla Talamo ${ }^{52}$, l'esistenza di modelli e cartoni, desunti dall' arte ellenistica ma di volta in volta -e forse localmente- adottati e rivisitati. Si delinea così un problema di utenza: i prodotti dovevano naturalmente soddisfare esigenze o possibilità diverse e gusti personali. Le differenti tipologie compositive trovano dunque spiegazione

${ }^{47}$ Per la documentazione delle gambe tornite desunte dalle pitture pompeiane cfr. l'accenno in Ransom 1905, p. 58 Tra gli altri casi basti pensare al fregio dionisiaco della Villa dei Misteri di Pompei, riconducibile al periodo del secondo stile, in cui l'eccezionale esecuzione del disegno pittorico si accompagna ad una conforme resa dei dettagli; qui in tre casi, sono ritratti, molto nitidamente, le gambe dei sedili su cui poggiano le figure. Nella scena del sacrificio è appena visibile l'elemento a campana del seggio, quasi interamente coperto da un drappo; mentre nella raffigurazione de lla toeletta sono minuziosamente ritratte le gambe dei sedili ornate da cinque coppie di anelli sovrapposti su di un terminale a campana; da ultimo la gamba del sontuoso seggio su cui è adagiata la figura isolata della domina, presenta una semplicità di linee; qui lo sviluppo tubolare della gamba viene interrotto da un proporzionata e sobria svasatura in alto, completata e conclusa allo stesso tempo dalla campana; in basso un altro elemento campaniforme è rifinito da una coppia di anelli.

48 D. M. Bailey, A Catalogue of the Lamps in the British Museum, 2, London 1980, tav. 19, Q 945, Q 946, Q 947, databili tra il 40 e l'80 d.C.; Giuliano, cit. (n. 28), pp. 210211, n. 12.

Letta 1984 , p. 78

50 Per un elenco dei letti con decorazione in avorio cfr. Letta 1984, pp. 95-97. Sui letti d'osso come diretta derivazione dei letti di tradizione greco-ellenistica cfr. Ransom e Richter 1966 cit. a nota 18; accenni anche in Nichols 1979, pp. 26-27, che vi riconosce una componente etrusca. Cfr. anche Letta 1984, p. 82 e sg.; Talamo, 1987-88, p. 72

51 R. Bianchi Bandinelli, cit. (n. 46), p. 202.

52 Talamo, 1987-88, p. 19.

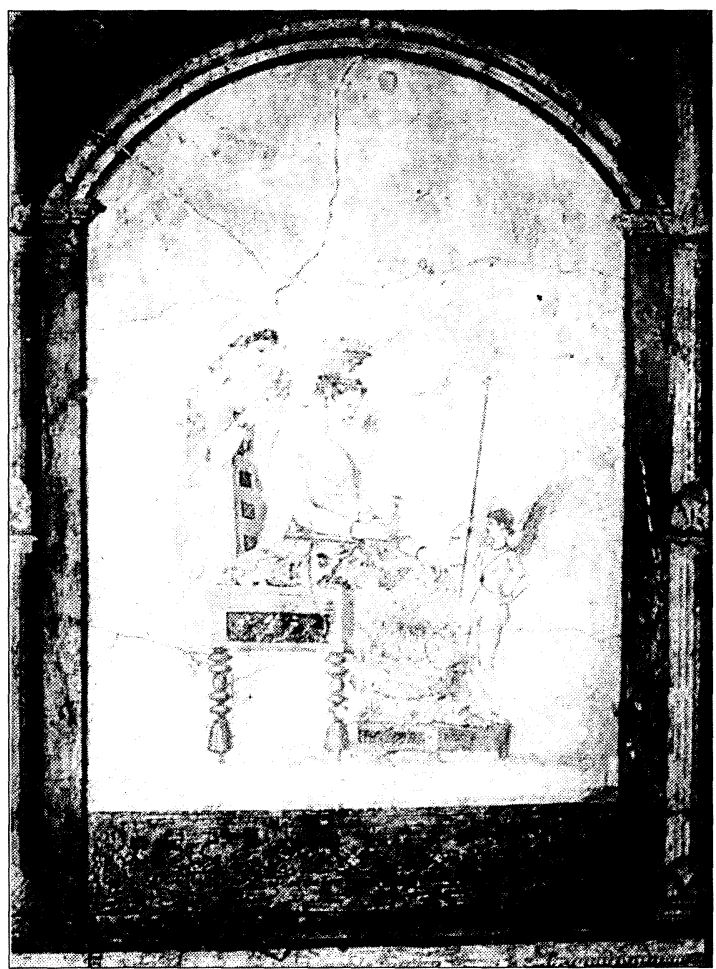

Fig. 25.-Afrodite in trono assistita da un'ancella con Eros dalla Villa Romana della Farnesina.

nel tipo di committenza, implicando direttamente l'esistenza di officine in grado di soddisfare le richieste di una clientela socialmente non omogenea.

Si pone dunque il problema dell'individuazione dei (o del) possibili centri di produzione. La costante presenza in area centro-italica dei letti funebri, fin

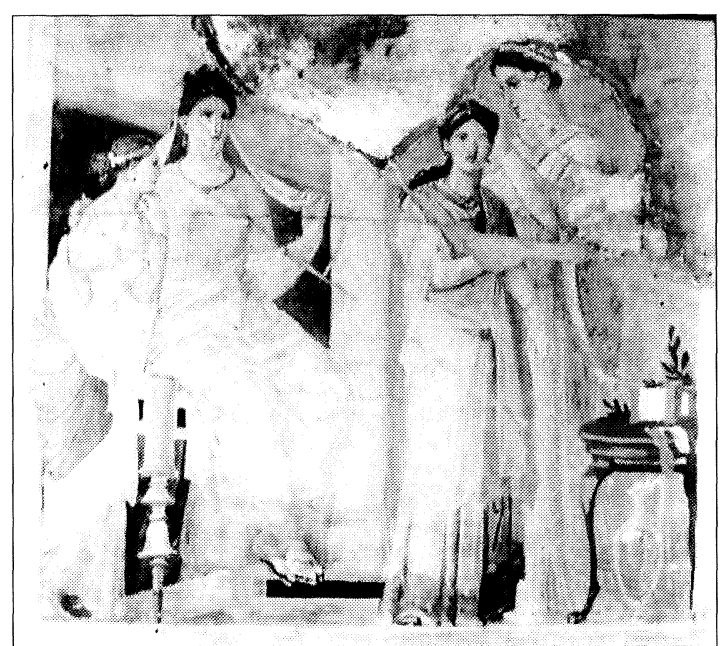

Fig. 26.- Scena di vestizione da Ercolano. Museo Nazionale di Napoli. 


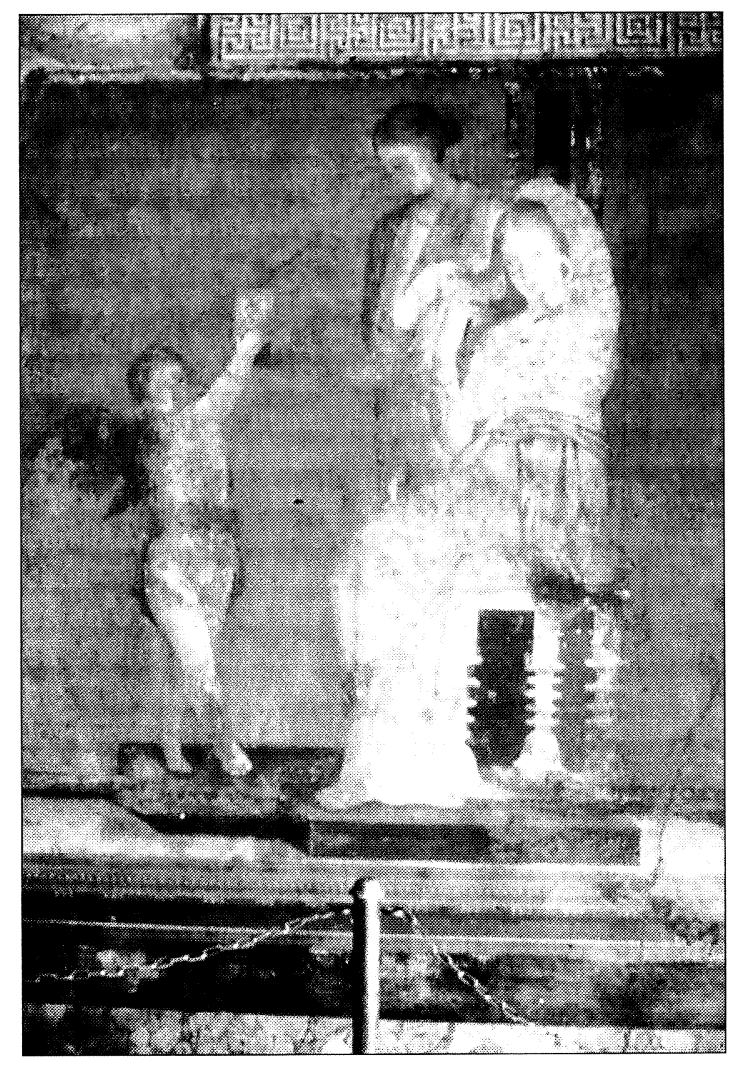

Fig. 27.-Pompei. Villa dei Misteri.

dal III-II secolo a.C. è chiaramente leggibile nella figura 29. Nei secoli successivi (figg. 30 e 31) il polo di diffusione sembra consolidarsi in Italia Centrale ed è dunque fortemente indiziario di un centro di produzione nella stessa area. L'importanza dei dati di distribuzione rilevabili dalla fig. 32 è però in parte inficiata dalla mancanza di precisi riferimenti cronologici. In effetti la continuità di rinvenimenti ad Ancona di letti databili in un periodo che va dalla metà del III secolo a. C. alla seconda metà del I secolo d.C., e l'esistenza di un gruppo di letti provenienti dall' area picena ${ }^{53}$, affini agli esemplari anconitani, hanno suggerito di identificare uno dei principali centri di produzione nella stessa Ancona, mentre l'Umbria, parte del Lazio e la Sabina dovevano essere serviti dalla zona di Norcia ${ }^{54}$. La concentrazione dei rinvenimenti nelle aree di queste

53 S. Vittore di Cingoli, Penne, Teramo, S. Lorenzo in Strada: cfr. Letta 1984 , p. 97 n. 35 ; p. 99 n. 50 ; p. 100 n. 72 ; Ortalli 1991, passim.

${ }^{54}$ Letta 1984, pp. 92-93; Nicholls, 1979, p. 26 propone l'Etruria come centro di produzione del letto di Cambridge. Ortalli (1991, 115-117) suggerisce la presenza di officine a nord delle Alpi, sviluppatesi fino alla fine del I - inizi II sec. d.C.

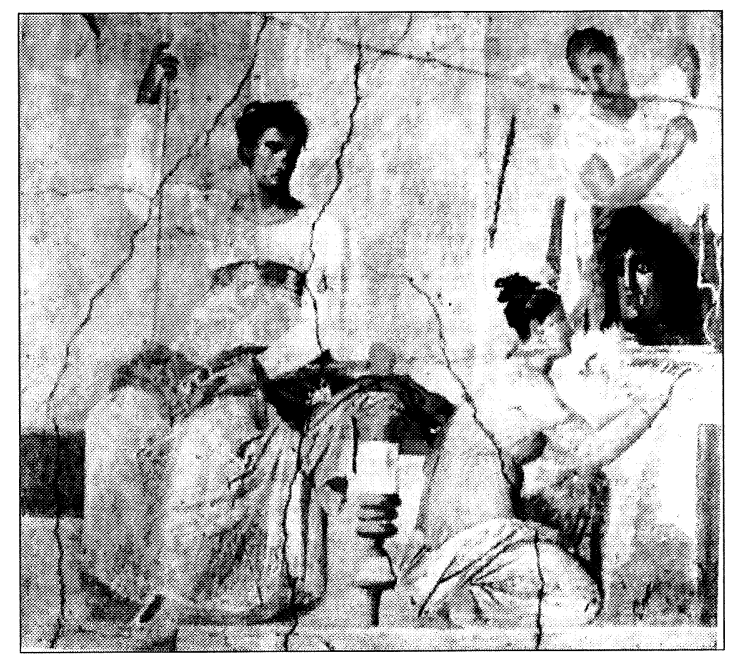

Fig. 28.-Attore re da Ercolano.

due città, nei secoli nei quali è minore la presenza dei letti funebri in territorio italico, potrebbe avvalorare questa ipotesi. Tuttavia da un'analisi comparata delle tavole di distribuzione, la zona in cui si evidenzia una maggiore concentrazione di ritrovamenti, accanto al territorio umbro, risulta essere quella del moderno Abruzzo.

Tralasciando in questa sede il problema, solo accennato, della individuazione delle officine, per la oggettiva mancanza di elementi determinanti, si può cautamente avanzare l'ipotesi che la precocità dell'apparizione di tale mobilia ad Ancona, scalo commerciale greco fin dal vi secolo a.C., sia da attribuire alle prime importazioni di questa classe di materiali dalla Grecia e non all'esistenza di un centro di produzione locale. L'apparizione dei letti nel territorio italico che, come abbiamo visto, non risale oltre la metà del III secolo a.C., suggerisce che l'impianto di una produzione locale sia stato preceduto da un periodo durante il quale il gusto per tali oggetti viene progressivamente assimilato determinandone l'affermazione sul mercato. In effetti è proprio tra la fine del II ed il I secolo a.C. che si evidenziano presenze significative dei letti con decorazione in osso nell' area umbro-abruzzese, le quali si generalizzano nei secoli successivi. E' dunque nel momento in cui la nuova moda si diffonde e non sono più sufficienti (o non più convenienti) le importazioni dalla Grecia, che si può collocare il sorgere delle prime officine destinate alla richiesta locale. Sembra dunque più opportuno parlare per il periodo iniziale di centri di importazione più che di produzione. Significativa, a questo proposito, è la presenza, nella stessa epoca, di rinvenimenti ad Ostia ed a Pompei, dove l'esistenza di contatti con 


\section{TAVOLA DI DISTRIBUZIONE}

Sulla base del catalogo del Letta, è stata resa graficamente e diacronicamente la distribuzione dei diversi tipi di letto, limitatamente alla distinzione tra letti con decorazione in avorio e letti con decorazione in osso.

Legenda: A, B, C, ecc. = la lettera indica la località letti con decorazione in avorio

$=$ letti con decorazione in osso

$=$ il numero indica la quantità di letti di quel tipo rinvenuti

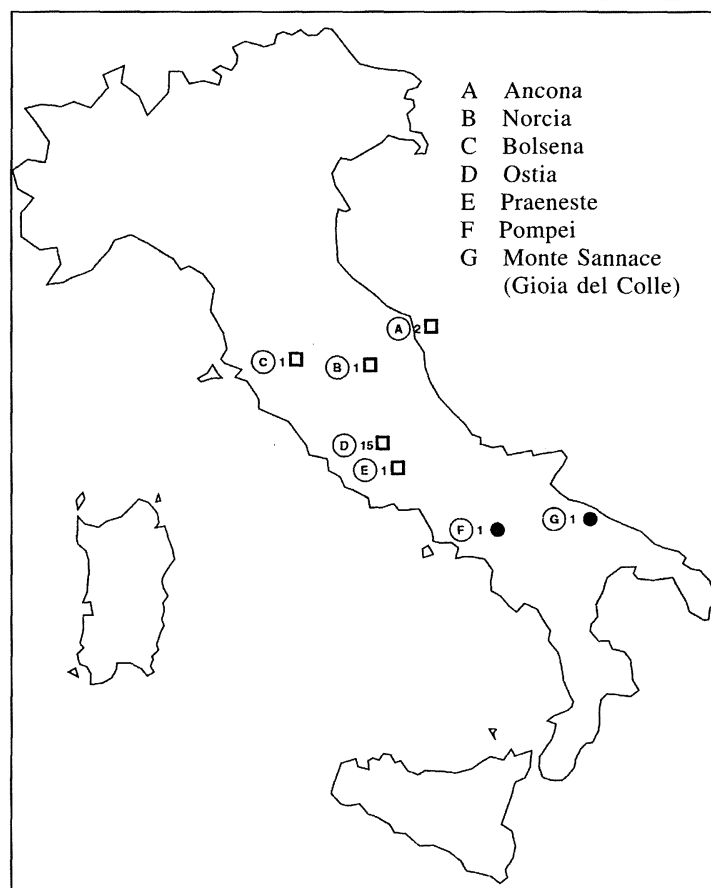

Fig. 29.-Area di diffusione seconda metà III-II sec. a.C.

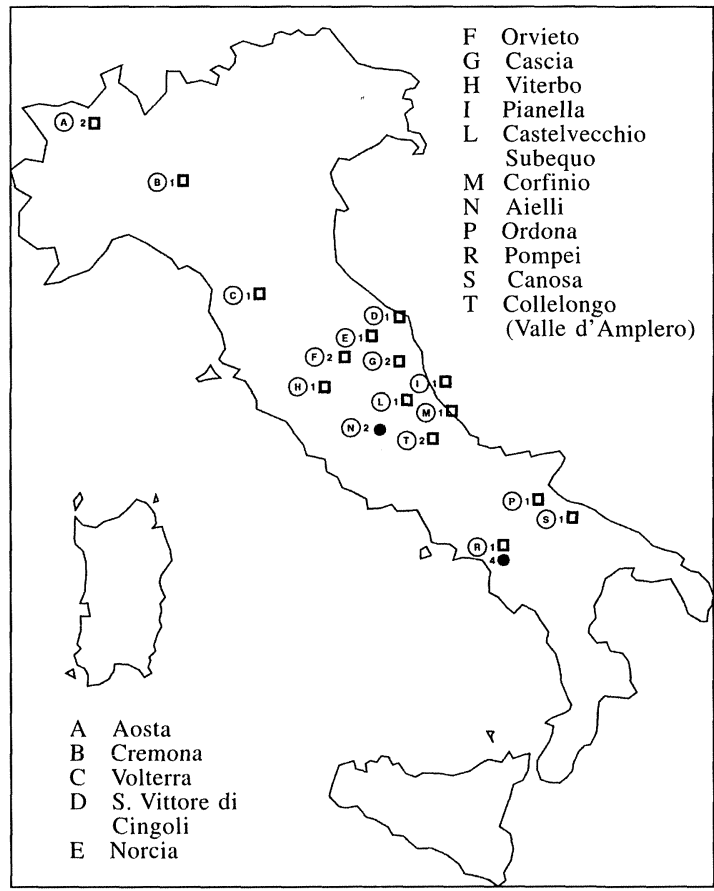

Fig. 31.-Area di diffusione fine I a.C.-inizi I d.C.

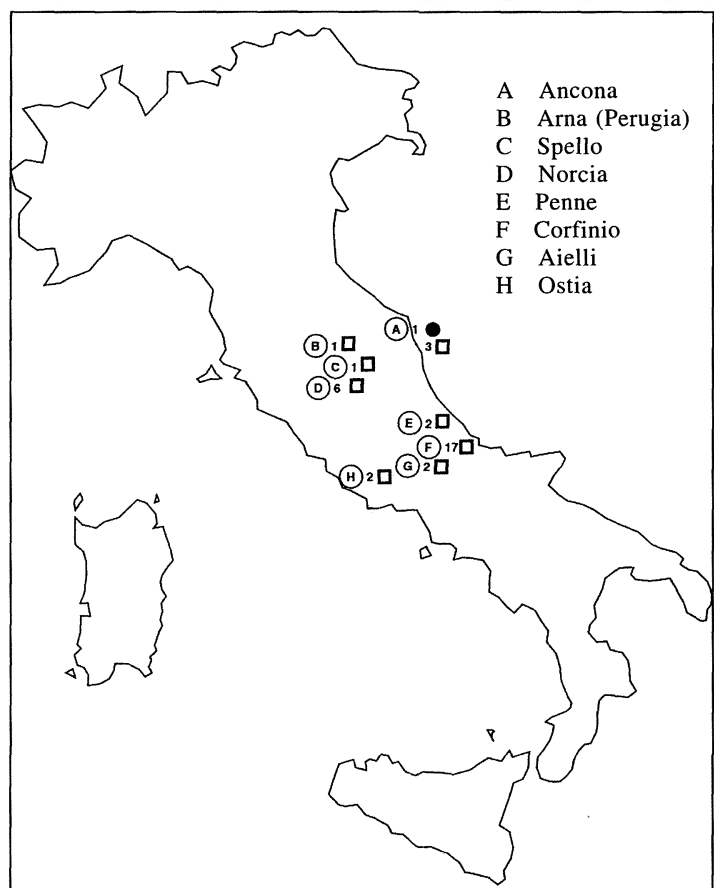

Fig. 30.-Area di diffusione fine II-I sec. a.C.

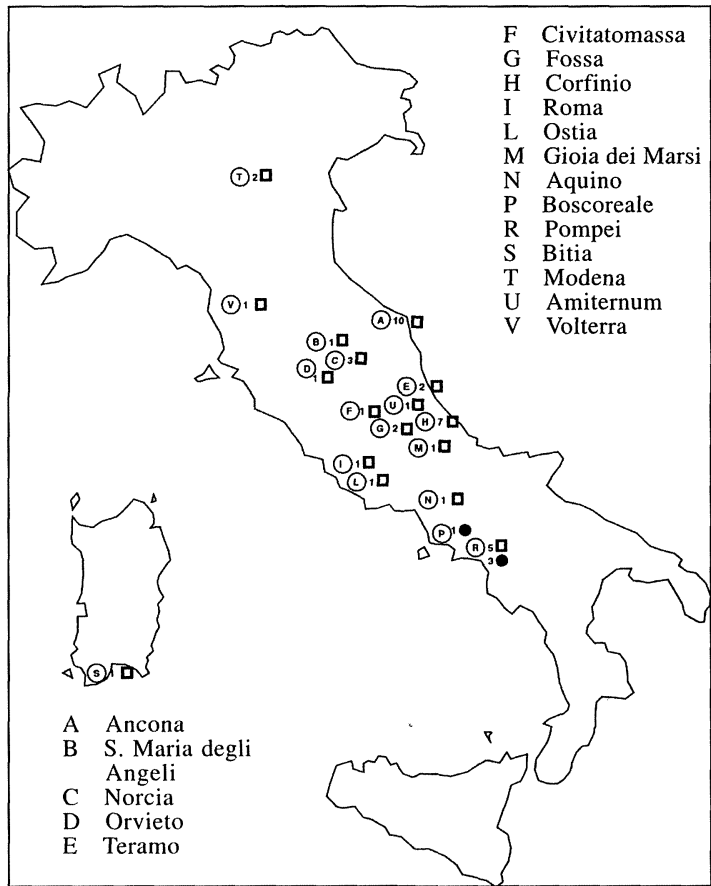

Fig. 32.-Area di diffusione dei letti non datati. 
il mondo ellenico è attestata da altre classi di materiali; da questi centri le vie di diffusione possono aver seguito gli stessi canali riconosciuti per gli altri prodotti.

Solo scarsi rinvenimenti -in totale 24- sono stati effettuati nelle province romane e per parte di essi sono incerti -o piuttosto vaghi- sia il luogo di provenienza che la datazione ${ }^{55}$. Sembra comunque di notare una buona percentuale di reperti databili nel I secolo d.C., quando anche è maggiore la diffusione nel territorio italiano. Tuttavia $\mathrm{i}$ dati in nostro possesso non sono ancora sufficienti per proporre un quadro significativo della diffusione dei letti funebri con decorazione in osso nel resto d'Europa e del bacino del Mediterraneo.

Il letto di Teramo-La Cona, consentendo un confronto cronologicamente definito, può divenire un utile punto di riferimento per i numerosi rinvenimenti decontestualizzati ${ }^{56}$.

${ }^{55}$ Letta 1984, pp. 95-111: per i frammenti in osso da modelli eburnei cfr. nn $37,39,43-45,52-55$; per i frammenti in osso da modelli bronzei cfr. nn 122-123, 125-126; per i frammenti in osso di incerta classificazione cfr. nn 177-186.

${ }_{56}$ L'unico precedente rinvenimento di un letto funebre effettuato a Teramo risulta disperso e non datato nel catalogo del Letta.

\section{BIBLIOGRAFIA}

BRIzIO, E., 1902: Ancona. Tombe dell'epoca romana, Notizie degli Scavi dell'Antichità $\quad(=N S c)$, 1902, pp. 445-459.

LETTA, C., 1984: Due letti funerari in osso dalla valle d'Amplero, Monumenti Antichi. Miscellanea III, 3, 1984, pp. 67-114.

Nicholls, R. V., 1979: A Roman Couch in Cambridge, Archaeologia or Miscellaneus Tracts relating to Antiquity, CVI, Oxford 1979, pp.1-32.

OrTALli, J., 1991: Un letto funerario in osso dalla necropoli di S. Lorenzo in Strada (Riccione). Note sul un prodotto di artigianato artistico norditalico, Studi Romagnoli XLII, 1991, pp. 101124.

PASQui, A., 1890: Di un antico letto di osso socoperto in una tomba di Norcia, Monumenti Accademia dei Lincei, I, 1890, pp. 241-256.

PASQUI, A., 1907: Tomba di età romana scoperta presso l'antica città di Amiternum, NSc 1907, pp. 145 ss.

Richter, G. M. A., 1966: The Furniture of the Greeks, Etruscans and Romans, London 1966 (2. ${ }^{a}$ ed.).

Talamo, E., 1987-88: Un letto funerario da una tomba dell'Esquilino, Bollettino della Commissione Archeologica di Roma XCII, 1987-88, pp. 17-102.

VAGLIERI, D.: NSc 1910, 551-552 e 1911, 44, 82 fig. 2. 